\title{
Editorial
}

\section{Advances in Matrices, Finite and Infinite, with Applications}

\author{
P. N. Shivakumar, ${ }^{1}$ Panayiotis Psarrakos, ${ }^{2}$ K. C. Sivakumar, ${ }^{3}$ and Yang Zhang ${ }^{1}$ \\ ${ }^{1}$ Department of Mathematics, University of Manitoba, Winnipeg, MB, Canada R3T 2N2 \\ ${ }^{2}$ Department of Mathematics, School of Applied Mathematical and Physical Sciences, \\ National Technical Univeristy of Athens, Zografou Campus, 15780 Athens, Greece \\ ${ }^{3}$ Department of Mathematics, Indian Institute of Technology Madras, Chennai 600 036, India \\ Correspondence should be addressed to P. N. Shivakumar; shivaku@cc.umanitoba.ca
}

Received 13 June 2013; Accepted 13 June 2013

Copyright (C) 2013 P. N. Shivakumar et al. This is an open access article distributed under the Creative Commons Attribution License, which permits unrestricted use, distribution, and reproduction in any medium, provided the original work is properly cited.

In the mathematical modeling of many problems, including, but not limited to, physical sciences, biological sciences, engineering, image processing, computer graphics, medical imaging, and even social sciences lately, solution methods most frequently involve matrix equations. In conjunction with powerful computing machines, complex numerical calculations employing matrix methods could be performed sometimes even in real time. It is well known that infinite matrices arise more naturally than finite matrices and have a colorful history in development from sequences, series and quadratic forms. Modern viewpoint considers infinite matrices more as operators defined between certain specific infinite-dimensional normed spaces, Banach spaces, or Hilbert spaces. Giant and rapid advancements in the theory and applications of finite matrices have been made over the past several decades. These developments have been well documented in many books, monographs, and research journals. The present topics of research include diagonally dominant matrices, their many extensions, inverse of matrices, their recursive computation, singular matrices, generalized inverses, inverse positive matrices with specific emphasis on their applications to economics like the Leontief input-output models, and use of finite difference and finite element methods in the solution of partial differential equations, perturbation methods, and eigenvalue problems of interest and importance to numerical analysts, statisticians, physical scientists, and engineers, to name a few.

The aim of this special issue is to announce certain recent advancements in matrices, finite and infinite, and their applications. For a review of infinite matrices and applications, see P. N. Shivakumar, and K. C. Sivakumar
(Linear Algebra Appl., 2009). Specifically, an example of an application can be found in the classical problem "shape of a drum," in P. N. Shivakumar, W. Yan, and Y. Zhang (WSES transactions in Mathematics, 2011). The focal themes of this special issue are inverse positive matrices including $M$ matrices, applications of operator theory, matrix perturbation theory, and pseudospectra, matrix functions and generalized eigenvalue problems and inverse problems including scattering and matrices over quaternions. In the following, we present a brief overview of a few of the central topics of this special issue.

For $M$-matrices, let us start by mentioning the most cited books by R. Berman and R. J. Plemmons (SIAM, 1994), and by R. A. Horn and C. R. Johnson (Cambridge, 1994), as excellent sources. One of the most important properties of invertible $M$-matrices is that their inverses are nonnegative. There are several works that have considered generalizations of some of the important properties of $M$-matrices. We only point out a few of them here. The article by D. Mishra and K. C. Sivakumar (Oper. Matrices, 2012), considers generalizations of inverse nonnegativity to the Moore-Penrose inverse, while more general classes of matrices are the objects of discussion in D. Mishra and K. C. Sivakumar (Linear Algebra Appl., 2012), and A. N. Sushama, K. Premakumari and K. C. Sivakumar (Electron. J. Linear Algebra 2012).

A brief description of the papers in the issue is as follows.

In the work of Z. Zhang, the problem of solving certain optimization problems on Hermitian matrix functions is studied. Specifically, the author considers the extremal inertias and ranks of a given function $f(X, Y): \mathbb{C}^{m \times n} \rightarrow$ $\mathbb{C}^{m \times n}$ (which is defined in terms of matrices $A, B, C$, and $D$ 
in $\left.\mathbb{C}^{m \times n}\right)$, subject to $X, Y$ satisfying certain matrix equations. As applications, the author presents necessary and sufficient conditions for $f$ to be positive definite, positive semidefinite, and so forth. As another application, the author presents a characterization for $f(X, Y)=0$, again, subject to $X, Y$ satisfying certain matrix equations.

The task of determining parametrized splitting preconditioners for certain generalized saddle point problems is undertaken by W.-H. Luo and T.-Z. Huang in their work. The well-known and very widely applicable Sherman-MorrisonWoodbury formula for the sum of matrices is used in determining a preconditioner for linear equations where the coefficient matrix may be singular and nonsymmetric. Illustrations of the procedure are provided for Stokes and Oseen problems.

The work reported by A. González, A. Suarez and D. Garcia deals with the problem of estimating the Frobenius condition of the matrix $A N$, given a matrix $A \in \mathbb{R}^{n \times n}$, where $N$ satisfies the condition that $N=\operatorname{argmin}_{X \in S}\|I-A X\|_{F}$. Here, $S$ is a certain subspace of $\mathbb{R}^{n}$ and $\|\cdot\|_{F}$ denotes the Frobenius norm. The authors derive certain spectral and geometrical properties of the preconditioner $N$ as above.

Let $B, C, D$ and $E$ be (not necessarily square) matrices with quaternion entries so that the matrix $M=\left(\begin{array}{ll}A & B \\ C & D\end{array}\right)$ is square. Y. Lin and Q.-W. Wang present necessary and sufficient conditions so that the top left block $A$ of $M$ exists satisfying the conditions that $M$ is nonsingular and the matrix $E$ is the top left block of $M^{-1}$. A general expression for such an $A$, when it exists, is obtained. A numerical illustration is presented.

For a complex hermitian $A$ of order $n$ with eigenvalues $\lambda_{1} \leq \lambda_{2} \leq \cdots \leq \lambda_{n}$, the celebrated Kantorovich inequality is $1 \leq x^{*} A x x^{*} A^{-1} x \leq\left(\lambda_{n}+\lambda_{1}\right)^{2} / 4 \lambda_{1} \lambda_{n}$, for all unit vectors $x \in$ $\mathbb{C}^{n}$. Equivalently $0 \leq x^{*} A x x^{*} A^{-1} x-1 \leq\left(\lambda_{n}-\lambda_{1}\right)^{2} / 4 \lambda_{1} \lambda_{n}$, for all unit vectors $x \in \mathbb{C}^{n}$. F. Chen, in his work on refinements of Kantorovich inequality for hermitian matrices, obtains some refinements of the second inequality, where the proofs use only elementary ideas (http://www.math.ntua.gr/ ppsarr/).

It is pertinent to point out that, among other things, an interesting generalization of $M$-matrices is obtained by $\mathrm{S}$. Jose and K. C. Sivakumar. The authors consider the problem of nonnegativity of the Moore-Penrose inverse of a perturbation of the form $A-X G Y^{T}$ given that $A^{\dagger} \geq 0$. Using a generalized version of the Sherman-Morrison-Woodbury formula, conditions for $\left(A-X G Y^{T}\right)^{\dagger}$ to be nonnegative are derived. Applications of the results are presented briefly. Iterative versions of the results are also studied.

It is well known that the concept of quaternions was introduced by Hamilton in 1843 and has played an important role in contemporary mathematics such as noncommutative algebra, analysis, and topology. Nowadays, quaternion matrices are widely and heavily used in computer science, quantum physics, altitude control, robotics, signal and colour image processing, and so on. Many questions can be reduced to quaternion matrices and solving quaternion matrix equations. This special issue has provided an excellent opportunity for researchers to report their recent results.
Let $\mathbb{H}^{n \times n}$ denote the space of all $n \times n$ matrices whose entries are quaternions. Let $Q \in \mathbb{U}^{n \times n}$ be Hermitian and selfinvertible. $X \in \mathbb{M}^{n \times n}$ is called reflexive (relative to $Q$ ) if $X=$ QXQ. The authors F.-L. Li, X.-Y. Hu, and L. Zhang present an efficient algorithm for finding the reflexive solution of the quaternion matrix equation: $A X B+C X^{*} D=F$. They also show that, given an appropriate initial matrix, their procedure yields the least reflexive solution with respect to the Frobenius norm.

In the work on the ranks of a constrained hermitian matrix expression, S. W. Yu gives formulas for the maximal and minimal ranks of the quaternion Hermitian matrix expression $C_{4}-A_{4} X A_{4}^{*}$, where $X$ is a Hermitian solution to quaternion matrix equations $A_{1} X=C_{1}, X B_{1}=C_{2}$, and $A_{3} X A_{3}^{*}=C_{3}$, and also applies this result to some special system of quaternion matrix equations.

Y. Yao reports his findings on the optimization on ranks and inertias of a quadratic hermitian matrix function. He presents one solution for optimization problems on the ranks and inertias of the quadratic Hermitian matrix function $Q-$ $X P X^{*}$ subject to a consistent system of matrix equations $A X=C$ and $X B=D$. As applications, he derives necessary and sufficient conditions for the solvability to the systems of matrix equations and matrix inequalities $A X=C, X B=D$, and $X P X^{*}=(>,<, \geq, \leq) Q$.

Let $R$ be a nontrivial real symmetric involution matrix. A complex matrix $A$ is called $R$-conjugate if $\bar{A}=R A R$. In the work on the hermitian $R$-conjugate solution of a system of matrix equations, C.-Z. Dong, Q.-W. Wang, and Y.-P. Zhang present necessary and sufficient conditions for the existence of the hermitian $R$-conjugate solution to the system of complex matrix equations $A X=C$ and $X B=D$. An expression of the Hermitian $R$-conjugate solution is also presented.

A perturbation analysis for the matrix equation $X-$ $\sum_{i=1}^{m} A_{i}^{*} X A_{i}+\sum_{j=1}^{n} B_{j}^{*} X B_{j}=I$ is undertaken by X.-F. Duan and Q.-W. Wang. They give a precise perturbation bound for a positive definite solution. A numerical example is presented to illustrate the sharpness of the perturbation bound.

Linear matrix equations and their optimal approximation problems have great applications in structural design, biology, statistics, control theory, and linear optimal control, and as a consequence, they have attracted the attention of several researchers for many decades. In the work of Q.-W. Wang and J. Yu, necessary and sufficient conditions of and the expressions for orthogonal solutions, symmetric orthogonal solutions, and skew-symmetric orthogonal solutions of the system of matrix equations $A X=B$ and $X C=D$ are derived. When the solvability conditions are not satisfied, the least squares symmetric orthogonal solutions and the least squares skew-symmetric orthogonal solutions are obtained. A methodology is provided to compute the least squares symmetric orthogonal solutions, and an illustrative example is given to illustrate the proposed algorithm.

Many real-world engineering systems are too complex to be defined in precise terms, and, in many matrix equations, some or all of the system parameters are vague or imprecise. Thus, solving a fuzzy matrix equation becomes important. In 
the work reported by X. Guo and D. Shang, the fuzzy matrix equation $\widetilde{A} \otimes \widetilde{X} \otimes \widetilde{B}=\widetilde{C}$ in which $\widetilde{A}, \widetilde{B}$, and $\widetilde{C}$ are $m \times m$, $n \times n$, and $m \times n$ nonnegative LR fuzzy numbers matrices, respectively, is investigated. This fuzzy matrix system, which has a wide use in control theory and control engineering, is extended into three crisp systems of linear matrix equations according to the arithmetic operations of LR fuzzy numbers. Based on the pseudoinverse matrix, a computing model to the positive fully fuzzy linear matrix equation is provided, and the fuzzy approximate solution of original fuzzy systems is obtained by solving the crisp linear matrix systems. In addition, the existence condition of nonnegative fuzzy solution is discussed, and numerical examples are given to illustrate the proposed method. Overall, the technique of LR fuzzy numbers and their operations appears to be a strong tool in investigating fully fuzzy matrix equations.

Left and right inverse eigenpairs problem arises in a natural way, when studying spectral perturbations of matrices and relative recursive problems. F.-L. Li, X.-Y. Hu, and L. Zhang consider in their work left and right inverse eigenpairs problem for $k$-hermitian matrices and its optimal approximate problem. The class of $k$-hermitian matrices contains hermitian and perhermitian matrices and has numerous applications in engineering and statistics. Based on the special properties of $k$-hermitian matrices and their left and right eigenpairs, an equivalent problem is obtained. Then, combining a new inner product of matrices, necessary and sufficient conditions for the solvability of the problem are given, and its general solutions are derived. Furthermore, the optimal approximate solution, a calculation procedure to compute the unique optimal approximation, and numerical experiments are provided.

Finally, let us point out a work on algebraic coding theory. Recall that a communication system, in which a receiver can verify the authenticity of a message sent by a group of senders, is referred to as a multisender authentication code. In the work reported by X. Wang, the author constructs multi-sender authentication codes using polynomials over finite fields. Here, certain important parameters and the probabilities of deceptions of these codes are also computed.

\title{
Acknowledgments
}

We wish to thank all the authors for their contributions and the reviewers for their valuable comments in bringing the special issue to fruition.

\author{
P. N. Shivakumar \\ Panayiotis Psarrakos \\ K. C. Sivakumar \\ Yang Zhang
}




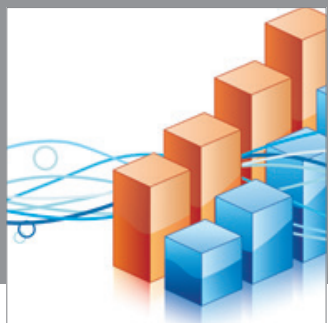

Advances in

Operations Research

mansans

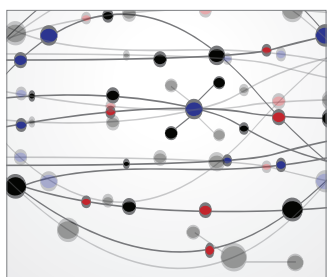

The Scientific World Journal
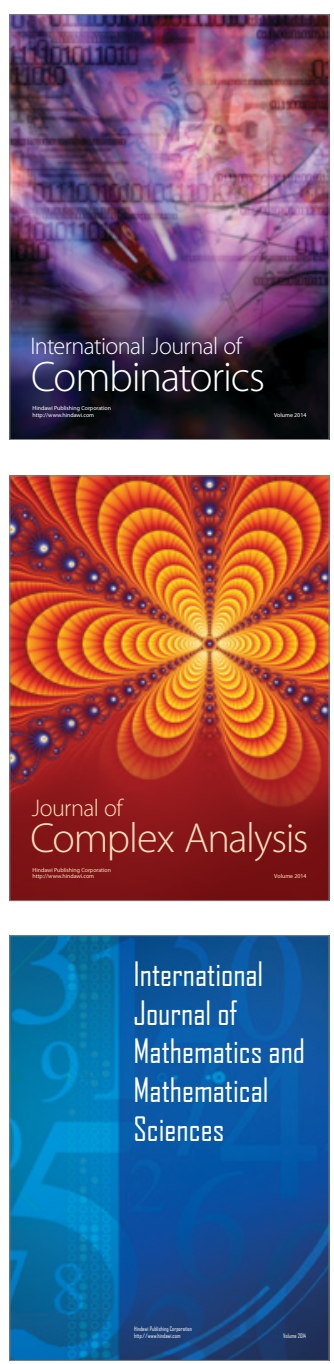
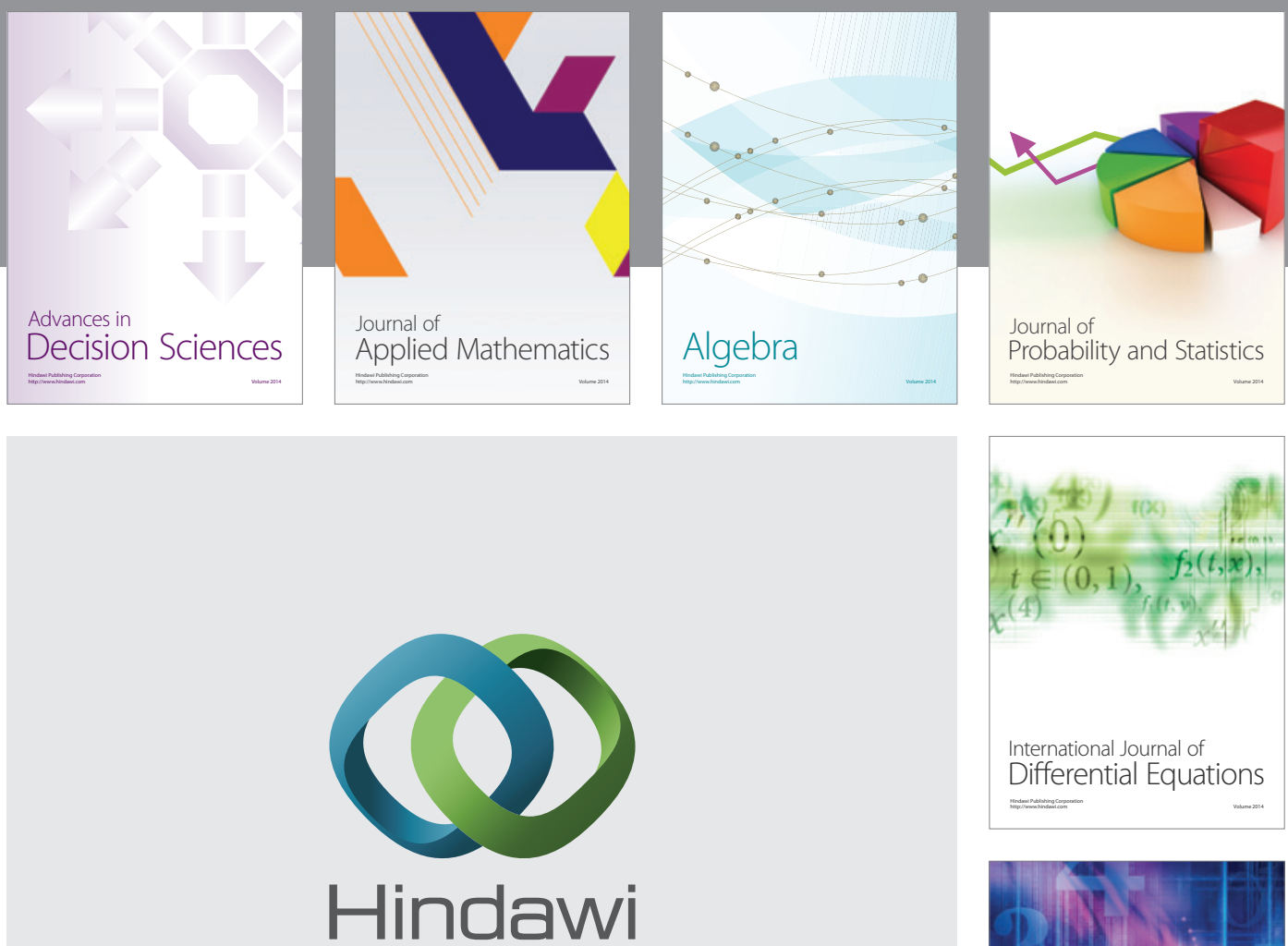

Submit your manuscripts at http://www.hindawi.com
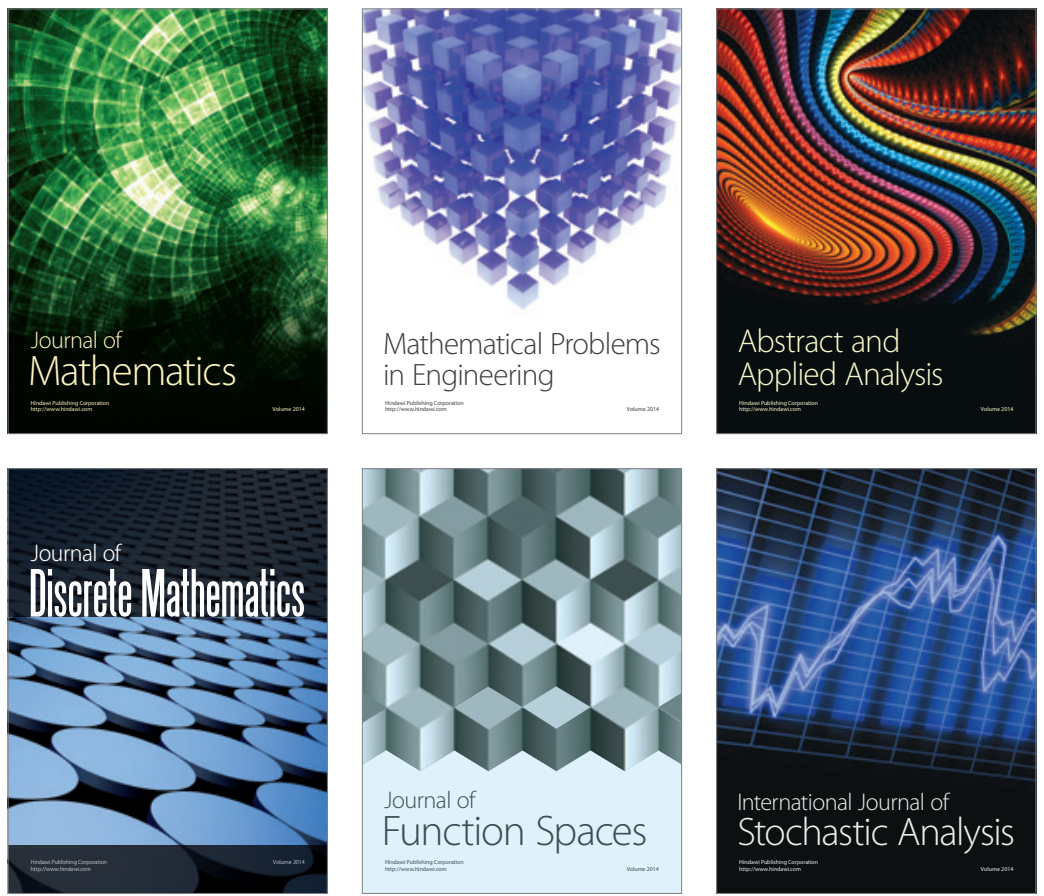

Journal of

Function Spaces

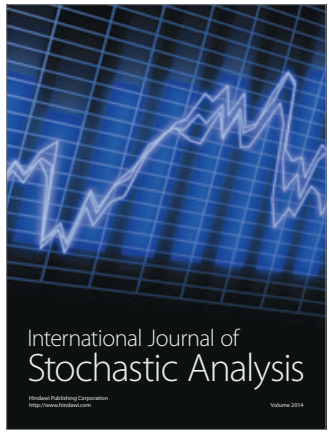

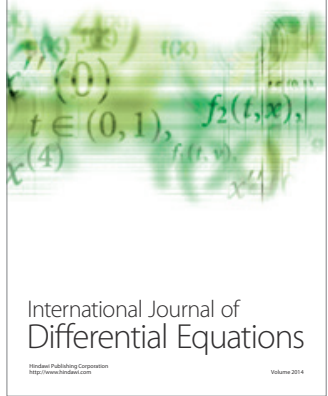
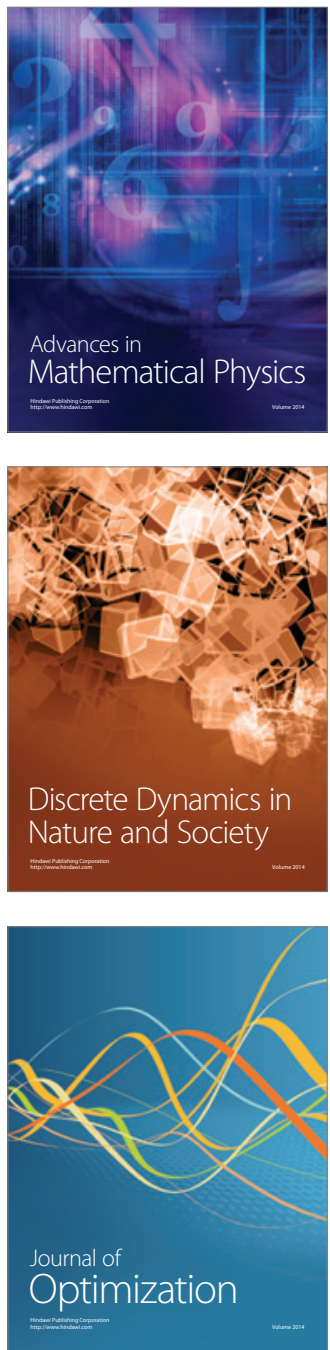